\title{
Task Performance is Prioritized Over Energy Reduction
}

\author{
Ravi Balasubramanian*, Member, IEEE, Robert D. Howe, Senior Member, IEEE, and Yoky \\ Matsuoka, Member, IEEE.
}

\begin{abstract}
The objective of this study was to characterize the temporal relationship between hand stiffness and task performance during adaptation to a brief contact task that required precision at the time of contact. The experiment required subjects to control the vertical position of a paddle on a computer display by grasping a robot's instrumented handle, with the goal of intercepting a virtual ball within $1 \mathrm{~mm}$ from the paddle center. A force transient was applied to the hand immediately after the ball-paddle impact to estimate the intrinsic hand impedance. There were two main results: (1) More trials were required for a brief contact task to find a low-energy strategy when compared with tasks that received feedback through the entire movement trajectory. (2) When the whole course of adaptation is long for brief contact tasks, viscoelastic forces were increased to achieve the task goal before the energy reduction initiated. Also, as the accuracy requirement was increased by changing the gain between handle and paddle motion through visual amplification, peak stiffness increased and occurred later, indicating that higher energy strategies are used for longer when the task's accuracy requirements were increased. These results indicated that task performance may be prioritized over energy reduction for a brief contact task.
\end{abstract}

\section{INTRODUCTION}

There are many tasks that require positional precision. Some tasks require accuracy for the whole movement path, while others require accuracy only at a particular point of the movement. For example, when hitting a volley in tennis, the path followed by the arm before the event is not as critical as the precision of position at the point of contact with the ball [36]. It has been shown that any task learning requires the central nervous system to identify the input-output characteristics of the motor system for that specific task [8,21,35]. Thus, in the tennis volleying example, the central nervous system has to learn to carefully modulate racquet position and stiffness to control ball trajectory after impact.

Manuscript prepared March 2008. This work was supported by grants ONR N00014-98-1-0669 and NIH 1 R21 EB005967. Asterisk indicates contact author

*R. Balasubramanian is with the Department of Computer Science and Engineering at the University of Washington, Seattle, WA (email: bravi@cs.washington.edu). R. D. Howe is with the Division of Engineering and Applied Sciences at Harvard University, Cambridge, MA. Y. Matsuoka is with the Department of Computer Science and Engineering at the University of Washington, Seattle, WA.

Copyright (C) 2008 IEEE. Personal use of this material is permitted. However, permission to use this material for any other purposes must be obtained from the IEEE by sending an email to pubspermissions@ieee.org.
In studying how the central nervous system learns tasks, it has been shown that viscoelastic forces through cocontraction at high energy cost are used early on in the learning process beyond what the task itself requires. With practice, the excess viscoelastic forces and the performance error are reduced over time to find a lowenergy strategy [30]. In some cases, it was noticed that limb stiffness was initially increased and then decreased over the course of learning a task [10], while error reduction followed an exponential decay $[6,7,9,11,17]$. The tasks used in the above studies required precision for the entire movement path while the subjects received feedback during the entire movement. In this study, we focused on a task that required positional precision only at a brief contact point in a movement without any feedback on the path followed to the goal. Such brief-contact tasks include drumming [15] and ball catching [23] which have been explored focusing on the role of impedance modulation to overcome bandwidth limitation. However, the time-history profiles of hand stiffness and task error during learning a brief-contact task have not been studied. Specifically, we were interested in understanding how the learning mechanisms changed when position accuracy was required only at a brief contact point when compared to the entire path and also if the learning process was longer. In order to answer these questions, the time-history profiles of hand stiffness and task error were evaluated simultaneously during a precision task with a brief contact. Portions of this work have been presented in [27].

\section{Materials And Methods}

\section{A. Apparatus}

A high-fidelity force-feedback haptic interface, the PHANTOM ${ }^{1}$, was used to estimate hand impedance (see Fig. 1a). The robot's standard stylus interface was replaced at the distal end with an instrumented handle mounted on a simple universal joint to allow natural hand position. The PHANTOM robot used in this experiment thus had three actuated rotational freedoms, two passive rotational freedoms at the universal joint, and three translational degrees of force feedback (peak forces up to $8.5 \mathrm{~N}$ could be exerted in any direction inside a $20 \mathrm{~cm}$ diameter workspace). Typical end-effector stiffness is $3.5 \mathrm{~N} / \mathrm{mm}$ and inertia $75 \mathrm{~g}$.

\footnotetext{
${ }^{1}$ Sensable Technologies Corporation; Premium Model 1.5
} 


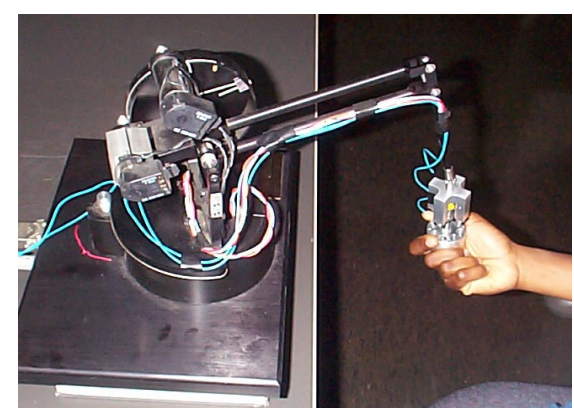

(a)

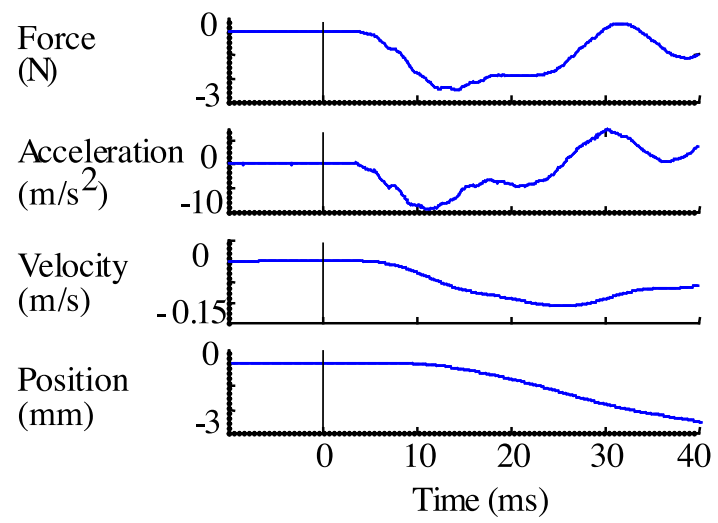

(c)

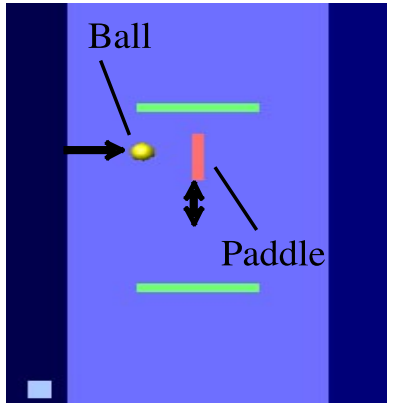

(b)

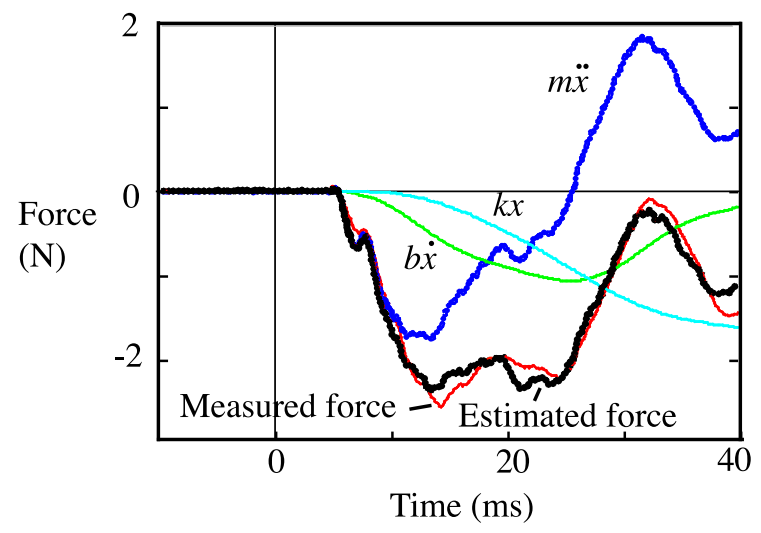

(d)

Fig. 1. The experimental set up includes (a) the Phantom haptic interface, an instrumented handle, and (b) a visual display showing the ball-paddle workspace. (c) The time profiles of force and paddle acceleration, velocity, and position during a typical trial. (d) A typical system identification plot showing measured (thin red line) and estimated forces (thick black line).

The instrumented handle included a piezoelectric accelerometer ${ }^{2}$ inside the handle and a piezoelectric force sensor $^{3}$ between the robot end point and the handle. The signals from these sensors were sampled at $10 \mathrm{kHz}$. The handle weighed $59 \mathrm{~g}$, of which $20 \mathrm{~g}$ was distal to the force sensor.

\section{B. Task and Protocol}

The task was to control the vertical position of a paddle displayed on a computer screen by grasping and moving the handle attached to the PHANTOM's distal end through wrist flexion and extension movements (see Fig.1b). The subject sat two feet in front of the computer monitor, and the $14 \mathrm{~mm}$ tall paddle was centered in a workspace limited by two vertical walls $60 \mathrm{~mm}$ apart. For each trial, a $4 \mathrm{~mm}$ diameter ball appeared at a random location on the left edge of the workspace and moved to the right at $30 \mathrm{~mm} / \mathrm{sec}$. Any effect from the subject's anticipation of the ball appearing was minimized by randomizing the time that it took for the next ball to appear (between 1-2 seconds).

The subject is instructed to intercept the ball at the center of the paddle by using the handle to move the

\footnotetext{
${ }^{2}$ Kistler Instrument Corporation; model 8630B50, sensitivity 101.5 $\mathrm{mV} / \mathrm{g}$.

${ }^{3}$ Kistler Instrument Corporation; model 9712A5, sensitivity 856 $\mathrm{mV} / \mathrm{lbf}$.
}

paddle vertically. If the contact between the ball and the paddle was within $1 \mathrm{~mm}$ of the paddle center, the ball turned green to indicate a successful action. If the ball did not contact the paddle within $1 \mathrm{~mm}$ of its center, the ball turned red to indicate failure. The duration between two tasks varied between five and six seconds.

Task performance was measured using the absolute vertical distance between the paddle's center point and the ball contact point (termed error). We also use "error at hand", obtained by dividing the visual error by the gain, to measure the error at the subject's hand. In both measures, smaller distance indicated better performance. To simulate ball contact, the robot delivered an impulse to the handle after the ball impacted the paddle $(50 \mathrm{~ms}$ after position error measurement). The subject's response to this impulse was used to estimate hand stiffness (see section II-C for more details). Note that the expectation of the impulse would have influenced the effective hand impedance when compared to the case where there is no disturbance. However, since the impulse was constant across trials, the effect on hand impedance was similar across trials, and the protocol measured only the relative change in hand impedance with change in accuracy requirement.

The subjects were instructed to avoid moving the paddle horizontally to hit the ball, but simply to align the 
vertical position with the ball and wait for the ball to hit the paddle. Each trial was also checked to confirm that the subject only moved the paddle vertically and that the subject's hand returned to the original horizontal location. In addition, the subjects's forearms were secured with a Velcro strap to the modified arm of the chair they sat on to eliminate motion at joints more distal to the handle than the wrist. The range of wrist motion was restricted using two visual markers placed $2 \mathrm{~cm}$ above and below the paddle on the monitor, corresponding to approximately half the wrist joint range at unity gain. The ball appeared only between these markers.

While previous work [10] has used "error at hand" to explore limb stiffness modulation, this study controls accuracy through visual amplification. Task accuracy requirement was modified by varying the gain between the handle's motion and the paddle's motion. Our experiment used gains of 1x, 8x, 16x, and 24x. For example, when the gain was set to $1 \mathrm{x}$, the virtual paddle moved the same distance on the computer screen that the handle moved in the subject's wrist. However, when the gain was set to 8 , the paddle moved eight times the physical distance traveled by the handle. Thus, compared to gain 1, the subject wrist had to move smaller distances with gain 8 . Increasing gains had the effect of increasing the accuracy required for the positioning task, and the visual display had sufficient resolution to be unaffected by gain changes.

Three groups containing six right-handed subjects each participated in the study, and each group underwent two sets of 250 trials each. Each group experienced gain $1 \mathrm{x}$ in set 1 , and, after a three-minute break, experienced an increased gain (one of $8 x, 16 x$, and 24x) in set 2. During the break, the subjects were informed that the sensitivity of the paddle movement might have changed, but were not told what the gain would be. Since each subject performed trials with gain $1 \mathrm{x}$ and a higher gain, the data from the gain $1 \mathrm{x}$ trials was used to normalize the subject's performance at the higher gain.

\section{System identification technique}

In the past, several limb-impedance identification strategies have been developed [1, 19, 20, 24, 25]. This paper estimated hand impedance using an impulse-based identification technique that allows instant parameter identification from one trial. When the ball contacted the paddle, the robot delivered a $3 \mathrm{~N}$ force impulse lasting $25 \mathrm{~ms}$ at its distal end (see Fig. 1c). The frequency of the impulse was constant across subjects and accuracy requirements. The time course of the impulse waveform was designed to avoid exciting the dominant vibration modes of the robot [16] and to provide a motion profile that facilitated the impedance estimation algorithm. The force was applied vertically to identify hand impedance in the direction of the hand's motion, rather than in the horizontal direction that the ball moved. The paddle was free to move in any direction in the workspace, and the impulse was the only force the handle applied. Since the impulse was applied $50 \mathrm{~ms}$ after position error measurement, the impulse did not affect the position error measurement.

The force direction was randomized between upward and downward to minimize bias from a voluntary or conditioned response. Some subjects missed hitting the ball entirely on the first trial in a session, and the impedance analysis was not conducted on the missed trial. No subjects missed beyond the first trial, and the adaptation trend was recorded without excluding or missing a trial for 250 consecutive trials. Note that friction forces were negligible (magnitude less than $0.04 \mathrm{~N}$ ). The visual scene updated at $30 \mathrm{~Hz}$, and the force command updated at $1 \mathrm{kHz}$.

The impedance estimation technique modeled hand impedance as a piecewise-linear, second-order mechanical system given by

$$
f(t)=m \ddot{x}(t)+b \dot{x}(t)+k\left(x(t)-x_{0}\right),
$$

where $f(t)$ represented the external force on the hand (from the robot handle) at time $t, x(t)$ the hand position, and $x_{0}$ the rest position of the spring element. The symbol $\dot{x}(t)$ represented hand velocity and $\ddot{x}(t)$ hand acceleration. The effective mass $m$, damping $b$, and stiffness $k$ are assumed to be constant for the duration of the impulse. This linear approximation is accurate for a fixed point over short durations [20]. Rather than identify the impedance of individual joints, this approach represented the hand-wrist combine in terms of lumped elements to focus on the impedance strategy employed during the task $[4,14,28]$. Note that the wrist-hand stiffness can be modulated by stiffness of the fingers which held the handle.

Starting from the onset of the force perturbation, the sensors in the PHANTOM handle provided hand acceleration measurements $(\ddot{x}(t))$ and force measurements $(f(t))$ at $10 \mathrm{kHz}$ for $40 \mathrm{msec}$. Velocity and position measurements (sub-micron accuracy) were computed by integrating the acceleration signal. In each trial, the paddle's rest position was set equal to the hand's position at the start of the force transient. We estimated the parameters $m, b$, and $k$ using least squared fits of (1) to the four hundred data points for each trial (see Fig. 1d for a typical result).

The parameter estimation technique was validated in the anticipated range of hand impedance by estimating the impedances of well-characterized metal springs and masses. Instead of the subject holding the robot handle, the springs and masses were coupled to the robot's handle. The mean errors in estimated mass and stiffness were less than $1 \%$, and the maximum errors in a single trial were $8.4 \%$ for mass and $3.7 \%$ for stiffness. Overall, there was excellent agreement (mean $r^{2}$ value was 0.988 ) between the measured force and the estimated force (back calculated using the estimated parameters $m, b$, and $k$ and the measured motion). Although the inertia and damping parameters were estimated as 
part of the system identification technique, the remainder of this study focused on stiffness modulation for two reasons. First, this study focused on the relationship between task performance and energy expenditure, and stiffness is directly correlated with energy expenditure. Second, the coupling between stiffness and damping has been shown to be weak in goal-directed voluntary movements and damping is modulated to a less extent than stiffness [3, 5, 29, 32].

In order to compare the time profiles of stiffness and task error across subjects and conditions, each set was normalized by its steady-state stiffness and error measurements. Thus, normalized stiffness $K_{1}(t)$ and error $E_{1}(t)$ were defined as follows:

$$
K_{1}(t)=K(t) / K_{s s}, E_{1}(t)=E(t) / E_{s s},
$$

where $K(t)$ was the trial's stiffness, $K_{s s}$ the set's steadystate stiffness, $E(t)$ the trial's error, and $e_{s s}$ the set's steady-state error.

To analyze of how a subject changed stiffness strategies and performance varied when compared with the subject's performance at gain $1 \mathrm{x}$, data from higher gain trials was normalized using steady-state stiffness and error measurements from the gain $1 \mathrm{x}$ trials. Thus, normalized stiffness $K_{2}(t)$ modulation and error $E_{2}(t)$ were defined as follows:

$$
K_{2}(t)=K(t) / K_{s s, 1 x}, E_{2}(t)=E(t) / E_{s s, 1 x} .
$$

where $K_{s s, 1 x}$ was the steady-state stiffness with gain $1 \mathrm{x}$ and $E_{s s, 1 x}$ the steady-state error with gain $1 \mathrm{x}$.

The variation in normalized stiffness (defined in (2) and (3)) with learning for each set was modeled as a double exponential curve to capture both the initial rise and the eventual decay of a set's stiffness estimates (similar to [10] where exponential curves were fitted to electromyography data). The least-square nonlinear fitted curve for each gain $(1,8,16$, or 24$)$ had the form:

$$
\begin{aligned}
K_{f i t}= & c_{k r}\left(1-e^{-(t-1) / r_{k r}}\right)-c_{k d}\left(1-e^{-(t-1) / r_{k d}}\right) \\
& +c_{k o},
\end{aligned}
$$

where $c_{k r}$ represented the constant multiplier of the initial exponential rise, $c_{k d}$ the constant multiplier of the eventual exponential decay, $r_{k r}$ the time-constant of exponential rise, and $r_{k d}$ the time-constant of exponential decay. The symbol $t$ represented the trial number, and $c_{k o}$ the stiffness at trial $t=1$.

Task performance was measured using error in positioning the paddle. The variation in normalized error (defined in (2) and (3)) in each set was modeled as an exponential decay with the form:

$$
E_{f i t}=c_{e d}\left(e^{-(t-1) / r_{e d}}\right)+c_{e o},
$$

where $c_{e d}$ represented the constant multiplier of the exponential decay, $c_{e r}$ the constant multiplier of the exponential rise, $r_{e d}$ the time constant for exponential decay, $r_{e r}$ the time constant for exponential rise, and $c_{e o}+c_{e d}$ the error at trial $t=1$. All significance tests were conducted using t-tests at $p=0.05$, and variation was represented by $95 \%$ confidence intervals and standard error.

Note that this experiment estimated stiffness during the static phase that followed vertical movements. [13] found that stiffness was correlated between the active motion phase and the immediate post-motion phase of a positioning task. While we did not measure the stiffness during the active motion phase, we expect it to be correlated to the post-motion phase stiffness because higher gains caused more positional inaccuracies in both active motion and post-motion phases.

\section{RESULTS}

\section{A. Stiffness and Error Trends During Adaptation}

The normalized stiffness $K_{1}(t)$ and error $E_{1}(t)$ measurements for all conditions along with a fitted doubleexponential curves are plotted in Fig. 2. The normalized data from different sets could be combined, given that fewer than ten percent of the 250 trials in a set were statistically different across gains. The fitted curve for stiffness had correlation coefficient 0.93 , and the fitted curve for error had correlation coefficient 0.79 . The trial at which normalized error $E_{1}(t)$ reached within $95 \%$ of the steady-state value $(29 \pm 5$ (rounded)) was close to the normalized stiffness $K_{1}(t)$ peak trial (36 \pm 4 (rounded)).

The normalized stiffness curve revealed that peak stiffness was significantly greater than trial 1 stiffness $\left(p<10^{-6}\right)$, stiffness peak value was significantly greater than steady-state stiffness $\left(p<10^{-5}\right)$, and steady-state stiffness was significantly greater than trial 1 stiffness $(p=0.002)$. In each set, the stiffness increase (time constant $27.1 \pm 4.5$ ) was quicker than the stiffness decrease (time constant $67.9 \pm 6.8$ ), and the rise time-constant was significantly less than the decay time-constant $\left(p<10^{-5}\right)$. However, the stiffness adaptation time constants were larger than those reported for adaptation to tasks that required precision for the entire movement path (mean stiffness rise time-constants range between 1 and 6 , and mean decay time-constants range between 17 and 30 [10]). Stiffness fluctuated (shown as confidence intervals in Fig. 2), but it was not correlated with unsigned wrist angles (correlation coefficient $0.15 \pm 0.03$ ). Interestingly, stiffness also did not correlate with the task performance of the previous trial (average absolute cross-correlation for any set was $0.11 \pm 0.01$ ), in contrast to [30].

The normalized error curve revealed that steady-state error was significantly smaller than trial 1 error $(p<$ $\left.10^{-5}\right)$. During adaptation, the error decreased even more rapidly (time constant $15.5 \pm 5.8$ ) than the stiffness increase $(p=0.06)$.

\section{B. Variation of Stiffness and Error Trends with Accuracy Requirement}

Fig. 3 shows the normalized stiffness $K_{2}(t)$ adaptation and error $E_{2}(t)$ reduction curves of gains $8 x$, 


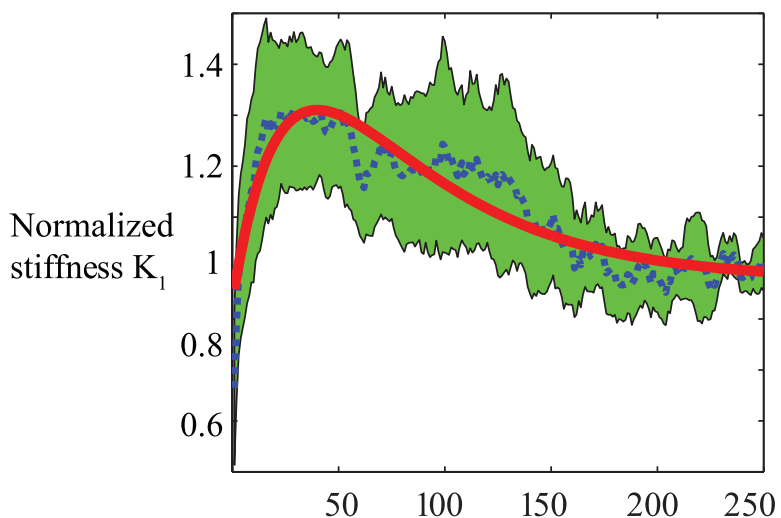

(a)

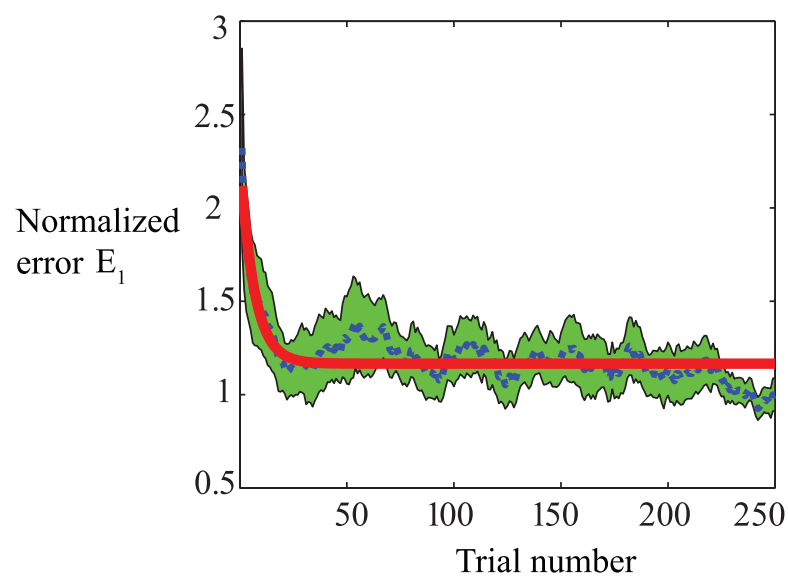

(b)

Fig. 2. (a) Normalized stiffness $K_{1}(t)$ and (b) normalized error $E_{1}(t)$ during a set of trials $(n=18)$. The bold solid curves (red) are functions fitted to the moving average plots (dashed blue line, window size $=$ 10). The green regions depict confidence intervals.

$16 x$, and 24x. Statistical analysis of normalized stiffness $K_{2}(t)$ across the conditions (see Table I) showed that peak stiffness with gain $1 \mathrm{x}$ was significantly less than peak stiffness with gains 16x and 24x. Also, steadystate stiffness with gain $1 \mathrm{x}$ was statistically different from steady-state stiffness with gains $8 x, 16 x$ and $24 x$. The peak-stiffness trial occurred later during adaptation as accuracy requirement increased (see Table I). In particular, the shift was significant in the cases of gain $1 \mathrm{x}$ versus $16 \mathrm{x}$ and $24 \mathrm{x}$ and gains $8 \mathrm{x}$ versus $24 \mathrm{x}$.

Statistical analysis of normalized error $E_{2}(t)$ showed that the steady-state errors increased as gain increased (see Table II; steady-state absolute error for gain 1x: $0.72 \pm 0.03 \mathrm{~mm})$. However, the error at hand (different from the visual error) was statistically smaller as accuracy requirement increased, and only the difference between error-at-hand for gains 16x and 24x was not statistically different. The trial at which error reached steady-state also appeared to occur later as accuracy requirement increased, as indicated by the fitted curves and the normalized data, but the shifts were not significant. Finally, error reached steady-state before the trial that stiffness peaked $(8 \mathrm{x}: p=0.09,16 \mathrm{x}$ and $24 \mathrm{x}$ : $p<0.05$ ).

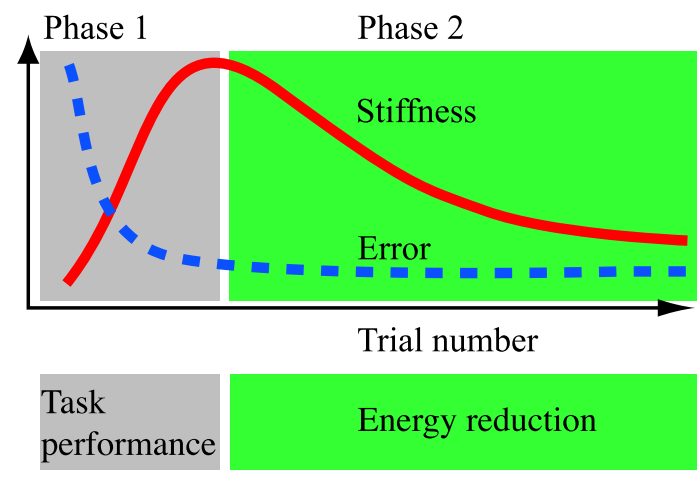

Phase 1

Phase 2

Fig. 4. Integration of viscoelasticity-dependent and energy-reduction control strategies during adaptation:(a) Stiffness adaptation and error reduction and (b) Contribution of viscoelasticity-dependent control and energy reduction-based control.

\section{Discussion}

The non-monotonic stiffness variation observed in this study is similar to the stiffness variation observed in $[10,13]$, and several studies have reported an exponential decay of error [7, 9, 17, 30]. What has not been observed previously was the temporal relationship between stiffness change and task performance error during a positional accuracy task. Specifically, it was observed in this study that the error reaching steady state triggered stiffness to stop increasing (as the stiffness peak occurred several trials after error plateaued) changing the nature of adaptation thereon. Thus, the adaptation time history was classified into two phases using this moment as the transition point: phase 1 occurred for the first several minutes where stiffness (or more generally viscoelastic forces) rapidly increased as error was rapidly decreased toward the task requirement level; and phase 2 occurred for a longer duration where stiffness decreased slowly while the error remained level.

The existence of phase 1 played an important role in the adaptation process. During phase 1, the subject gained practice with the task. However, energy reduction was not given higher priority over task performance. Phase 2, however, was a period where energy reduction was the primary goal, even while error was not reduced further. These observations are consistent with the hypothesis of adaptive internal model-based control [12, $21,35]$, where subjects build internal or feedforward models to improve task performance while reducing energy consumption. While it was unclear if task performance was prioritized over energy reduction (through reduced viscoelastic contribution) in [30] (since performance improvement and energy reduction occurred simultaneously from the beginning of the session), a modified learning process where phase 1 was primarily an error-reduction phase (with little priority given to energy reduction) was inferred in this study (see Fig. 4). This can be inferred from the increased involvement of stiffness-based control and poor correlation between the task success or failure and next-trial stiffness. 


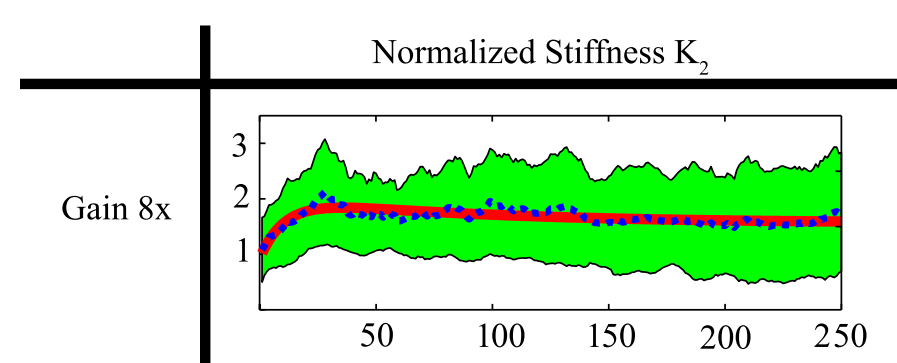

(a)

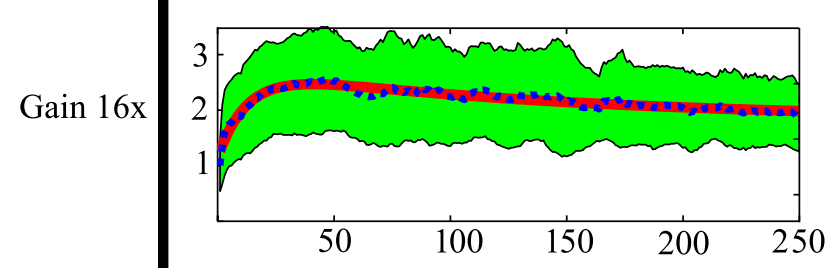

(b)

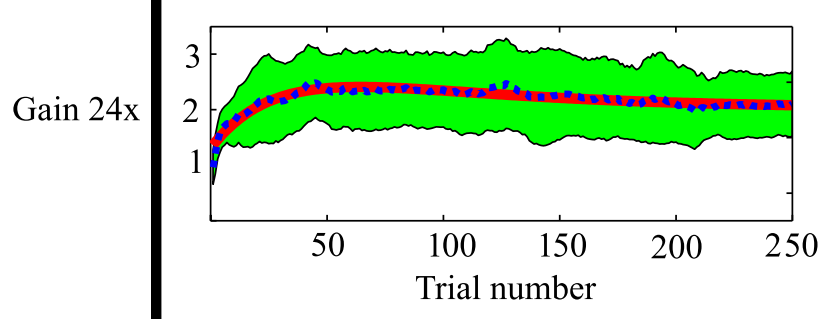

(c)
Normalized Error $\mathrm{E}_{2}$

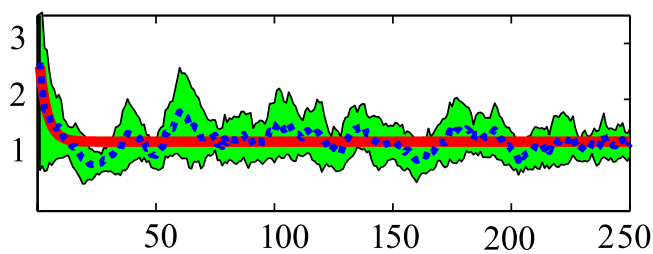

(d)

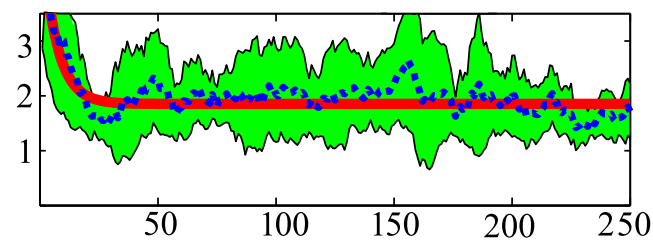

(e)

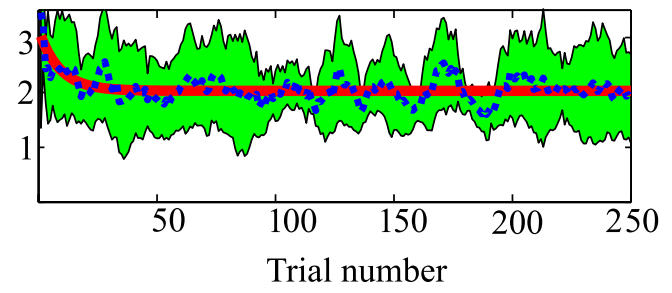

(f)

Fig. 3. (a), (b), (c) Normalized stiffness $K_{2}(t)$ normalized by steady-state stiffness at gain 1x. (d), (e), (f) Error estimates $E_{2}(t)$ normalized by steady-state stiffness at gain 1x. Each plot has solid (red) lines which represent fitted curves and dotted (blue) lines which represent moving average $($ window size $=10)$ plots. Green regions depict confidence intervals $(n=6)$.

TABLE I

NORMALIZED STIFFNESS $K_{2}$ VARIATION AS A FUNCTION OF ACCURACY REQUIREMENT

\begin{tabular}{|c|c|c|c|c|c|}
\hline \multirow[t]{2}{*}{ Gain } & \multirow{2}{*}{$\begin{array}{l}\text { Correlation } \\
\text { Coefficient }\end{array}$} & \multicolumn{2}{|c|}{ Peak Trial } & \multirow{2}{*}{$\begin{array}{l}\text { Peak } \\
\text { Value }\end{array}$} & \multirow{2}{*}{$\begin{array}{c}\text { Steady State } \\
\text { Value }\end{array}$} \\
\hline & & Fitted curve & Data (rounded) & & \\
\hline $1 \mathrm{x}$ & 0.92 & 32 & $25(5)^{f g}$ & $1.37(0.14)^{a b}$ & $1.00(0)^{c d e}$ \\
\hline $8 \mathrm{x}$ & 0.75 & 36 & $34(5)^{h}$ & $1.9(0.42)$ & $1.63(0.54)^{c}$ \\
\hline $16 \mathrm{x}$ & 0.95 & 41 & $45(7)^{f}$ & $2.5(0.46)^{a}$ & $1.95(0.32)^{d}$ \\
\hline $24 x$ & 0.94 & 64 & $57(4)^{g h}$ & $2.35(0.36)^{b}$ & $2.07(0.29)^{e}$ \\
\hline
\end{tabular}

Superscripts $a-h$ denote significant difference.

Numbers in brackets are standard errors.

TABLE II

NORMALIZED ERROR $E_{2}$ VARIATION AS A FUNCTION OF ACCURACY REQUIREMENT

\begin{tabular}{c|c|c|c|c|c}
\hline Gain & Correlation & \multicolumn{2}{|c|}{ Reach Steady-State Trial } & \multicolumn{2}{|c}{ Steady State } \\
& Coefficient & Fitted Curve & Data & Value (Visual) & Value (Hand) \\
\hline \hline $1 \mathrm{x}$ & 0.73 & - & - & $1.00(0.00)^{\text {pij }}$ & $1(0.00)^{\text {klm }}$ \\
$8 \mathrm{x}$ & 0.65 & 11 & $22(7)$ & $1.19(0.12)^{p q r}$ & $0.15(0.01)^{k n o}$ \\
$16 \mathrm{x}$ & 0.84 & 21 & $22(5)$ & $1.58(0.14)^{i q}$ & $0.1(0.01)^{l n}$ \\
$24 \mathrm{x}$ & 0.57 & 28 & $26(5)$ & $2.04(0.40)^{j r}$ & $0.09(0.02)^{\text {mo }}$ \\
\hline \hline
\end{tabular}

Superscripts $i-r$ denote significant difference.

Numbers in brackets are standard errors. 
When the accuracy requirement increased, the peakstiffness trial occurred later relative to the trial at which the error plateaued. Furthermore, the peak stiffness values increased as the accuracy requirement increased. This indicates that when the task was harder to accomplish, phase 1 became longer and utilized more vigorous viscoelastic forces until the task was accomplished. This was supported by the fact that the steady-state raw error value reached the task goal (error less than $1 \mathrm{~mm}$ ) at the end of phase 1 (raw error $0.81 \pm 0.03 \mathrm{~mm}$ for gains $1 \mathrm{x}$, $8 \mathrm{x}$, and $16 \mathrm{x}$ combined).

However, this strategy was not as effective for gain $24 \mathrm{x}$ where the task goal could not be achieved at its steady state (raw error $1.33 \pm 0.08 \mathrm{~mm}$ ). The peak stiffness for gain $24 \mathrm{x}$ was no different from the peak stiffness for gain 16x, while the time between when the performance error reached its steady state and the stiffness peaked was longer than for the other gains where the task goal was achieved. This means that if the task goal was not reached, the viscoelastic forces kept increasing its effort longer before moving onto phase 2 . The shift to phase 2 may be caused by fatigue or other biomechanical factors that made it difficult to raise the stiffness any higher for this task. It was the accuracy requirement that made the task difficult to achieve rather than the stiffness limit, since the error had plateaued much earlier than the stiffness peak.

An interesting aspect of error adaptation was observed in the transition period from phase 1 and phase 2 . In the case of gains $8 x$ and $16 x$, it was noticed that error during the transition period was significantly less than error during subsequent trials $\left(p<10^{-3}\right)$. Specifically, after error decreased rapidly during phase 1 , it was noticed that error decreased below the steady-state value during the transition period before rising to the steadystate value. It appeared that after phase 1 , the central nervous system may have compromised on error in order to reduce viscoelastic forces before settling into a steadystate error.

So what was different with this study's task that it showed two different phases, an error reduction phase and an energy reduction phase? The task here involved small movements with a requirement to be accurate for a brief period of time for each trial. The movement was similar to moving a computer mouse for a small distance and clicking a small button accurately, or moving the arm in a sport like tennis where arm position at the time of contact must be accurate. The small movements and brief contact made it difficult to quickly find a low-energy strategy that satisfied the energy requirement, given that delayed, error-based feedback motor responses generated by reflex circuits have been hypothesized as the template for internal model adaptation [22, 34, 35]. For a task that provided only a small amount of sensory feedback, it was possible that phase 1 with its high-energy strategy was needed to achieve this task within a reasonable time. This was supported by the fact that the stiffness adaptation time constant was much larger for our study than what were previously reported for larger arm movements [13, $17,35]$.

It was noticed that normalized steady-state stiffness for gain $1 \mathrm{x}$ was significantly less than normalized steadystate stiffness for gains $8 x, 16 x$, and 24x (see Table I), while normalized peak stiffness for gain $1 \mathrm{x}$ was significantly less than normalized peak stiffness for gains $16 \mathrm{x}$ and $24 \mathrm{x}$. This matches with previous work [31-33], where subjects performed a positioning task with elbow rotations and used increased stiffness as the target circle size decreased. In general, this finding of increased stiffness with greater accuracy requirement correlated with Hogan's theory of robotic manipulation and impedance control [18]. According to this theory, while a robotic controller typically uses position or force to optimize the task execution, tasks involving accurate interaction between physical systems, such as a hand manipulating a rubber ball, required careful impedance modulation in response to a motion imposed by the environment. Furthermore, the optimal impedance is specified by the task and the environment. The results from this present study implied that even though the task was to improve positional accuracy, the central nervous system controlled performance through impedance.

There are several daily-life tasks that involve precision positioning, and these can cause inordinate stresses on the human body particularly when they become repetitive. For example, tasks such as using the computer mouse or keyboard, precise assembly, performing musical instruments, and video games involve significant use of the wrist and fingers. Similar to the accuracy requirement we used in our experiment, computer users or game players could set a "pointer speed" for the computer mouse that controlled the proportionality between hand motion and monitor-cursor motion, and some keyboards for handheld devices could be displayed at different sizes. Our results suggested that the learning period increased as accuracy requirement increased, as in the case of computer displays with increased pointer speed or keyboards with smaller keys. Coupled with the high grip force required with increased gain level, both transiently and after adaptation, this posed an increasing risk of upper-extremity musculoskeletal disorders such as wrist injuries and carpal tunnel syndrome [2, 26]. This suggested that more detailed studies of hand stiffness adaptation linked with computer and device design are worth pursuing to minimize the risk of injury.

\section{REFERENCES}

[1] G. C. Agarwal and G. Gottlieb. Oscillation of the human ankle joint in response to applied sinusoidal torque on the foot. J Physiol, 268(1):151-76, June 1977.

[2] T. J. Armstrong, B. J. Martin, A. Franzblau, D. M. Rempel, and P. W. Johnson. Mouse input devices and work-related upper limb disorders. Working With Display Units 94, Elsevier Science, pages 375-380, 1995. 
[3] D. J. Bennett. Stretch reflex responses in the human elbow joint during a voluntary movement. J Physiol, 474:339-351, 1994.

[4] D. J. Bennett, J. M. Hollerbach, Y. Xu, and I. W. Hunter. Time-varying stiffness of human elbow joint during cyclic voluntary movement. Exp Brain Res, 88:433-442, 1992.

[5] D. J. Bennett, J. M. Hollerbach, Y. Xu, and I. W. Hunter. Time-varying stiffness of human elbow joint during cyclic voluntary movement. Exp Brain Res, 88:433-442, 1992.

[6] E. Burdet, D. W. Franklin, R. Osu, K. P. Tee, M. Kawato, and T. E. Milner. How are internal models of unstable tasks formed? In Proc. of the 26th Ann. Int. Conf. of the IEEE EMBS, pages 4491-4494, 2004.

[7] E. Burdet, R. Osu, D. W. Franklin, T. E. Milner, and M. Kawato. The central nervous system stabilizes unstable dynamics by learning optimal impedance. Nature, 414:446-449, 2001.

[8] M. Darainy, N. Malfait, P. L. Gribble, F. Towhidkhah, and D. J. Ostry. Learning to control arm stiffness under static conditions. J Neurophysiol, pages 3344-3350, 2004.

[9] J. R. Flanagan, E. Nakano, H. Imamizu, R. Osu, T. Yoshioka, and M. Kawato. Composition and decomposition of internal models in motor learning under altered kinematic and dynamic environments. J Neurosci, 19(20):RC34, 1999.

[10] D. W. Franklin, R. Osu, E. Burdet, M. Kawato, and T. E. Milner. Adaptation to stable and unstable dynamics achieved by combined impedance control and inverse dynamics model. J Neurophysiol, 90:3270-3282, 2003.

[11] D. W. Franklin, U. So, M. Kawato, and T. E. Milner. Impedance control balances stability with metabolically costly muscle activation. J Neurophysiol, 92:30973105, 2004.

[12] F. Gandolfo, F. A. Mussa-Ivaldi, and E. Bizzi. Motor learning by field approximation. The Proceedings of the National Academy of Sciences USA., 93(9):3843-3846, 1996.

[13] P. L. Gribble, L. I. Mullin, N. Cothros, and A. Mattar. A role of cocontraction in arm movement accuracy. J Neurophysiol, 89(5):2396-2405, 2003.

[14] A. Z. Hajian and R. D. Howe. Identification of the mechanical impedance at the human fingertip. ASME J Biomechanical Engineering, 119(1):109114, 1997.

[15] A. Z. Hajian, D. S. Sanchez, and R. D. Howe. Drum roll: Increasing bandwidth through passive impedance modulation. In Proc. of the Int. Conf. on Robotics and Automation, pages 2294-2299, 1997.

[16] K. L. Hillsley and S. Yurkovich. Vibration control of a two-link flexible robot arm. Dynamics and Control, 3(3):261-280, 1993.

[17] M. R. Hinder and T. E. Milner. Novel strategies in feedforward adaptation to a position-dependent perturbation. Exp Brain Res, 165:239-49, 2005.

[18] N. Hogan. The mechanics of multi-joint posture and movement control. Biol Cybern, 52:315-331, 1985.

[19] I. W. Hunter and R. E. Kearney. Dynamics of human angle stiffness: variation with mean angle torque. J Biomech, 15(10):747-752, 1982.

[20] I. W. Hunter and R. E. Kearney. System identification of human joint dynamics. Critical Rev. in Biomed. Engr., 18(1):55-87, 1990.

[21] M. Kawato. Internal models for motor control and trajectory planning. Curr. Opinion in Neurobiol., 9:718-727, 1999.

[22] M. Kawato, K. Furukawa, and R. Suzuki. A hierarchical neural-network model for control and learning of voluntary movement. Biol Cybern, 57(3):169-185, 1987.

[23] F. Lacquaniti, N. A. Borghese, and M. Carrozzo. Internal models of limb geometry in the control of hand compliance. J. Neurosci, 12(5):1750-1762, 1992.

[24] F. Lacquaniti, M. Carrozzo, and N. A. Borghese. Time-varying mechanical behavior of multijointed arm in man. J Neurophysiol, 69(3):1443-1464, 1993.

[25] J. B. MacNeil and R. E. Kearney. Identification of time-varying biological systems from ensemble data. IEEE Trans. on Biomed Engr., 39(12):12131225, 1992.

[26] L. Mani and F. Gerr. Work-related upper extremity musculoskeletal disorders. Primary Care, 27(4):845-864, 2000.

[27] Y. Matsuoka and R. D. Howe. Hand impedance change during learning of a novel contact task. In World Cong. on Med. Phys. and Biomed. Engr., 2000.

[28] T. E. Milner. Dependence of elbow viscoelastic behavior on speed and loading in voluntary movements. Exp Brain Res, 93:177-180, 1993.

[29] T. E. Milner and C. Cloutier. Damping of the wrist joint during voluntary movement. Exp Brain Res, 122:309-317, 1998.

[30] R. Osu, D. W. Franklin, H. Kato, H. Gomi, K. Domen, T. Yoshioka, and M. Kawato. Shortand long-term changes in joint co-contraction associated with motor learning as revealed from surface emg. J Neurophysiol, 2002.

[31] R. Osu, N. Kamimura, H. Iwasaki, E. Nakano, C. M. Harris, Y. Wada, and M. Kawato. Optimal impedance control for task achievement in the presence of signal-dependent noise. J Neurophysiol, 92:1199-1215, 2004.

[32] L. P. J. Selen, P. J. Beek, and J. H. van Dieen. Impedance is modulated to meet accuracy demands during goal-directed arm movements. Exp Brain Res, 172:129-138, 2006.

[33] L. P. J. Selen, J. H. van Dieen, and P. J. Beek. Impedance modulation and feedback corrections in 
tracking targets of variable size and frequency. $J$ Neurophysiol, 96:2750-2759, 1996.

[34] S. Stroeve. Learning combined feedback and feedforward control of a musculoskeletal system. Biol Cybern, 75(1):73-83, 1996.

[35] K. A. Thoroughman and R. Shadmehr. Electromyographic correlates of learning an internal model of reaching movements. J Neurosci, 9(19):8573-88, 1999.

[36] E. Todorov and M. I. Jordan. Optimal feedback control as a theory of motor coordination. Nature Neurosci, 5(11):1226-1235, 2002.

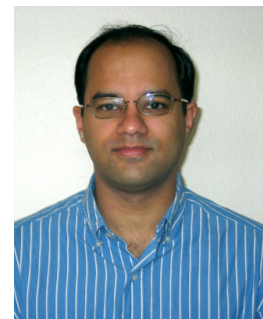

Ravi Balasubramanian Ravi Balasubramanian received the B.Eng. (1999) and M.Eng. (2000) degrees in mechanical engineering from the National University of Singapore and a Ph.D. in robotics (2006) from Carnegie Mellon University. His doctoral work on a novel locomotion technique called legless locomotion received the best student paper nomination at the IEEE International Conference on Robotics and $\mathrm{Au}$ tomation in 2004. Dr. Balasubramanian is currently a post-doctoral research associate at the University of Washington, Seattle, exploring the learning mechanisms of the central nervous system and the control redundancy in the human hand though human studies and anatomically correct robotic hands.

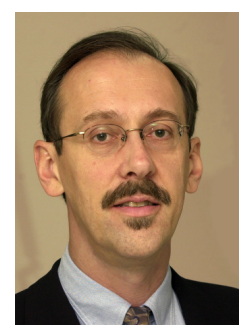

Robert D. Howe Robert D. Howe is Gordon McKay Professor of Engineering in the Harvard School of Engineering and Applied Sciences. Dr. Howe earned a bachelors degree in physics from Reed College, then worked as a design engineer in the electronics industry in Silicon Valley. He received a doctoral degree in mechanical engineering from Stanford University in 1990, and then joined the faculty at Harvard. Dr. Howe founded the Harvard BioRobotics Laboratory, which investigates the roles of sensing and mechanical design in motor control, in both humans and robots. His research interests focus on manipulation, the sense of touch, and human-machine interfaces. Biomedical applications of this work include the development of robotic and image-guided approaches to minimally invasive surgical procedures.

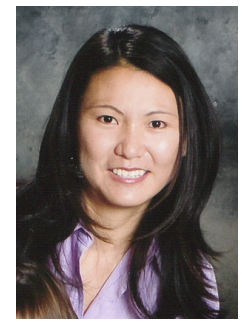

Yoky Matsuoka Yoky Matsuoka received the B.S. in electrical engineering and computer science (EECS) from the University of California, Berkeley, in 1993 and the M.S. and $\mathrm{Ph} . \mathrm{D}$. in EECS from the Massachusetts Institute of Technology (MIT) in 1995 and 1998, respectively. She was a Postdoctoral Fellow in the Brain and Cognitive Sciences Department at MIT and in Mechanical Engineering at Harvard University. She is currently Associate Professor in the Computer Science and Engineering at the University of Washington, Seattle. Prof. Matsuoka received the Presidential Early Career Award for Scientists and Engineers (PECASE) in 2004, the IEEE Robotics and Automation Society Early Academic Career Award in 2005, and MacArthur Fellowship in 2007. 